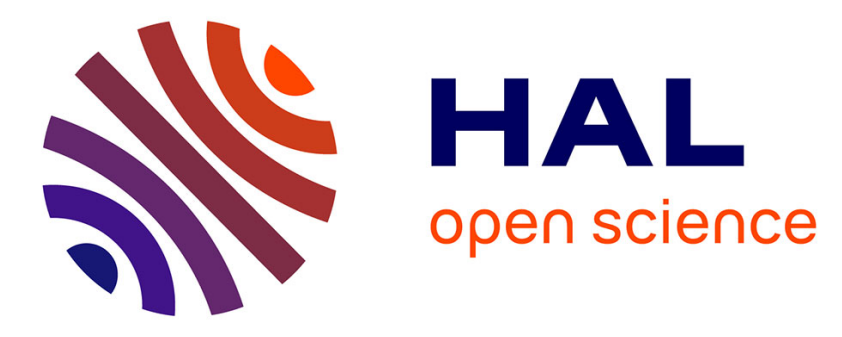

\title{
Mode-Stirring Impact in Radar Cross Section Evaluation in Reverberation Chamber
}

Ariston Reis, Francois Sarrazin, Elodie Richalot, Philippe Pouliguen

\section{To cite this version:}

Ariston Reis, Francois Sarrazin, Elodie Richalot, Philippe Pouliguen. Mode-Stirring Impact in Radar Cross Section Evaluation in Reverberation Chamber. 2018 International Symposium on Electromagnetic Compatibility - EMC EUROPE, Aug 2018, Amsterdam, France. 10.1109/EMCEurope.2018.8485023 . hal-01894044

\section{HAL Id: hal-01894044 https://hal.science/hal-01894044}

Submitted on 18 Oct 2018

HAL is a multi-disciplinary open access archive for the deposit and dissemination of scientific research documents, whether they are published or not. The documents may come from teaching and research institutions in France or abroad, or from public or private research centers.
L'archive ouverte pluridisciplinaire HAL, est destinée au dépôt et à la diffusion de documents scientifiques de niveau recherche, publiés ou non, émanant des établissements d'enseignement et de recherche français ou étrangers, des laboratoires publics ou privés. 


\section{Mode-Stirring Impact in Radar Cross Section Evaluation in Reverberation Chamber}

\author{
Ariston Reis, François Sarrazin, Elodie Richalot \\ Université Paris-Est, ESYCOM (EA2552), \\ UPEMLV, ESIEE-Paris, CNAM \\ F-77454 Marne-la-Vallée, France \\ ariston.defreitastavaresdosreis@u-pem.fr
}

\begin{abstract}
This paper presents the evaluation of the Radar Cross Section (RCS) pattern of a rectangular metallic plate from Reverberation Chamber measurement. It is seen that the measured RCS strongly depends on the environment and especially on the stirrer position. An approach based on averaging the measurement over a stirrer rotation is presented and allows increasing the measurement accuracy when compared to simulated data.
\end{abstract}

Keywords-Reverberation Chamber, Radar Cross Section.

\section{INTRODUCTION}

Radar Cross Section (RCS) is the commonly used parameter to describe the scattering behavior of an object. It is of interest in a wide range of topics including stealth optimization, radar detection, RFID interrogation [1] and antenna characterization [2]. Its determination is usually

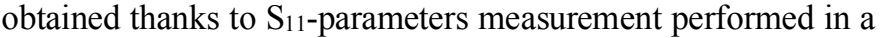
large anechoic chamber. It requires an accurate calibration of the measurement setup using a well-known canonical target such as a sphere, as well as a fine time-windowing implying Fourier transformations and also a hardware time-gating. Recently, Reverberation Chambers (RCs) have been investigated to measure the RCS of metallic objects. Indeed, the statistically uniform and isotropic incident field within the usable volume is very suitable to obtain the Absorption Cross Section, as well as the total RCS [3]. However, the monostatic or bistatic RCS retrieval within an RC is not as obvious. Based on earlier works on antenna directivity pattern measurement [4], authors in [5] proposed a novel approach to retrieve the ballistic wave between an antenna and a scattering object within a diffuse environment such as an RC, which enables RCS pattern measurement. This method presents the advantages to avoid any Fourier transformations as well as to use a cheaper measurement setup compared to classical anechoic chamber measurement.

The considered method is a two steps process that requires

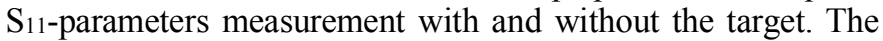
difference between the two then leads to the object RCS though a regression process. Thus, the RCS accuracy depends on the overall Signal-to-Noise Ratio (SNR). In [5], the mode stirrer is kept in a fixed position while in this paper, we suggest taking advantages of the stirring process to average the measurement over a stirrer rotation and thus lower the overall measurement noise.

\author{
Philippe Pouliguen \\ Strategy Directorate \\ Direction Generale de l'Armement (DGA) \\ Paris 75509, France \\ philippe.pouliguen@intradef.gouv.fr
}

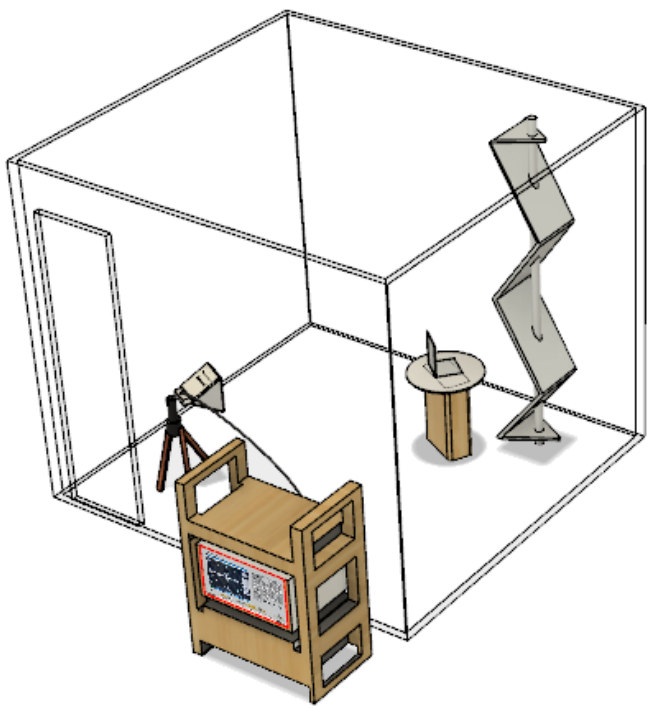

Fig. 1. Radar Cross Section measurement setup in Reverberation Chamber.

This paper is constructed as follows: Section II presents the measurement process details. Then, the RCS extraction procedure is exposed in Section III. Measurement results compared to simulation ones in Section IV clearly show the contribution of mechanical stirring to RCS measurement accuracy.

\section{MEASUREMENT PROCESS}

\section{A. Measurement setup}

For this measurement, a horn-antenna is placed within the working volume of the $\mathrm{RC}$ and connected to a port of a vector network analyzer (VNA) Rohde\&Schwarz ZNB8. The rectangular metallic plate (of dimensions $20 \mathrm{~cm}$ horizontally and $25 \mathrm{~cm}$ vertically), which represents the target, is positioned on a cardboard box at a distance $R=2.29 \mathrm{~m}$ from the antenna and is centered at the same height (Fig.1). As the chosen frequency band for this measurement is $6 \mathrm{GHz}-6.4 \mathrm{GHz}$, this distance $\mathrm{R}$ is large enough so that the ballistic wave can be considered as spherical on the target. It has to be noticed that the horn antenna, the rectangular plate and the mode stirrer are aligned. To evaluate the RCS pattern in the azimuth plane, the target is rotated around the vertical axis from -30 to 30 degrees with a 1 degree step. The horn-antenna is vertically polarized 
with an electric field along the height of the RC. The considered frequency range is very high compared to the RC Lowest Useable Frequency (around $400 \mathrm{MHz}$ ), so that the field within the cavity can be considered as isotropic and uniform.

\section{B. Measurement in the empty Reverberation Chamber}

During this measurement without the target (but with its support), the $\mathrm{RC}$ is excited by the horn-antenna at a frequency $f_{0}$ in the 6-6.4 GHz frequency range. The purpose of this first measurement is to determine the response of the cavity itself. Hence, the scattering parameter $S\left(f_{0}\right)$ is measured by the VNA (once calibrated at the antenna's connector level) over a stirrer rotation (with a rotation step of $30^{\circ}$ ). It can be expressed as follows [5]:

$$
S\left(f_{0}\right)=S_{F S}\left(f_{0}\right)+\left(1-\left|S_{F S}\left(f_{0}\right)\right|^{2}\right) H\left(f_{0}\right) \eta_{\text {ant }}
$$

In this equation, the term $S_{F S}\left(f_{0}\right)$ represents the reflection coefficient of the antenna in free space (it can be found by calculating the mean of the reflection coefficient over a stirrer rotation in the empty $\mathrm{RC}$. The second term is composed by the expression $\left(1-\left|S_{F S}\left(f_{0}\right)\right|^{2}\right)$ that represents the losses due to antenna mismatch, the antenna radiation efficiency $\eta_{\text {ant }}$ corresponding to the losses related to antenna performance, and $H\left(f_{0}\right)$ that represents the chamber transfer function at the antenna location and is supposed to follow a normal Gaussian distribution versus frequency as well as stirrer position in a well-operating RC.

\section{Measurement of the target backscattering}

Measurements are then performed after inserting the target. For each position of the target, the reflection coefficient is measured over a stirrer rotation (with a $30^{\circ}$ rotation step). When the RC is loaded by a target, the previous equation for the reflection coefficient is changed with the appearance of the term $C\left(f_{0}\right) \sqrt{\sigma^{T}\left(f_{0}\right)}$ :

$$
\begin{gathered}
S^{T}\left(f_{0}\right)=S_{F S}\left(f_{0}\right)+C\left(f_{0}\right) \sqrt{\sigma^{T}\left(f_{0}\right)}+ \\
\left(1-\left|S_{F S}\left(f_{0}\right)\right|^{2}\right) H^{T}\left(f_{0}\right) \eta_{\text {ant }}
\end{gathered}
$$

This additional term represents the backscattering by the target of the ballistic wave towards the antenna. In particular, the complex quantity $C\left(f_{0}\right)$ describes the wave propagation forwards from the antenna to the target and backwards from the target to the antenna at the frequency $f_{0}$. The term $\sigma^{T}$ stands for the Radar Cross Section of the target at the same frequency $f_{0}$. As the distance between the antenna and the target is sufficient for the ballistic wave to be considered as almost plane at the target level, the amplitude of $C\left(f_{0}\right)$ can be simplified as:

$$
\left|C\left(f_{0}\right)\right|=\frac{G_{\text {ant }}\left(f_{0}\right) \lambda_{0}}{(4 \pi)^{3 / 2 R^{2}}}\left(1-\left|S_{F S}\left(f_{0}\right)\right|^{2}\right)
$$

We can notice that this term is proportional to antenna gain $G_{a n t}$, and inversely proportional to the square distance $R$ between antenna and target.
As the dimension of the target is much smaller than the distance $\mathrm{R}$, the phase is considered as constant along the target and this leads to the following simplified expression:

$$
C\left(f_{0}\right)=\left|C\left(f_{0}\right)\right| \cdot \exp \frac{-j 2 \pi f_{0} 2 R}{c} \cdot \exp \left(j \phi_{0}\right)
$$

with $\phi_{0}$ an arbitrary constant phase. The phase variation corresponds to a wave propagation at the speed of light $c$ along the path $2 R$ forwards and backwards between the measuring antenna and the target.

\section{Radar Cross Section retrieval}

To obtain the expression that permits to extract the RCS, we compute the difference between the two previous measurements:

$$
\begin{gathered}
S^{T}\left(f_{0}\right)-S\left(f_{0}\right)=\left(1-\left|S_{F S}\left(f_{0}\right)\right|^{2}\right)\left(H^{T}\left(f_{0}\right)-H\left(f_{0}\right)\right) \eta_{\text {ant }} \\
+\sqrt{\sigma^{T}\left(f_{0}\right)} \frac{G_{\text {ant }}\left(f_{0}\right) \lambda_{0}}{(4 \pi)^{3 / 2 R^{2}}}\left(1-\left|S_{F S}\left(f_{0}\right)\right|^{2}\right) \\
\times \exp \frac{-j 2 \pi f_{0} 2 R}{c} \cdot \exp \left(j \phi_{0}\right)
\end{gathered}
$$

This equation (5) consists in two terms. The first one is proportional to $H^{T}\left(f_{0}\right)-H\left(f_{0}\right)$ that is assumed to be a random variable following a centered Gaussian distribution (when the frequency or the stirrer position varies) as each term of this difference does. This assumption will permit to consider this term as an additive random noise $N(f)$ and to extract the RCS from the second one. The latter contains the magnitude of the backscattered signal from the target. If we consider either its real or imaginary part, this term varies as a sine wave versus frequency. Thus, we can write:

$$
\begin{gathered}
\operatorname{Re}\left(S^{T}\left(f_{0}\right)-S\left(f_{0}\right)\right)= \\
\sqrt{\sigma^{T}\left(f_{0}\right)} \frac{G_{\text {ant }}\left(f_{0}\right) \lambda_{0}}{(4 \pi)^{3 / 2 R^{2}}}\left(1-\left|S_{F S}\left(f_{0}\right)\right|^{2}\right) \\
\times \cos \left(\frac{2 \pi f_{0} 2 R}{c}-\phi_{0}\right)+N(f)
\end{gathered}
$$

Simplifying this equation, it becomes:

$$
\operatorname{Re}\left(S^{T}\left(f_{0}\right)-S\left(f_{0}\right)\right)=A \times \cos \left(\frac{2 \pi f_{0} 2 R}{c}-\phi_{0}\right)+N(f)(7)
$$

where $A$ stands for the amplitude of the signal and is calculated while minimizing the following expression:

$$
A \cos \left(\frac{2 \pi f_{0} 2 R}{c}-\phi_{0}\right)-\operatorname{Re}\left(S^{T}\left(f_{0}\right)-S\left(f_{0}\right)\right)
$$

Once $A$ is found, the RCS can be deduced as the other coefficients are known. 


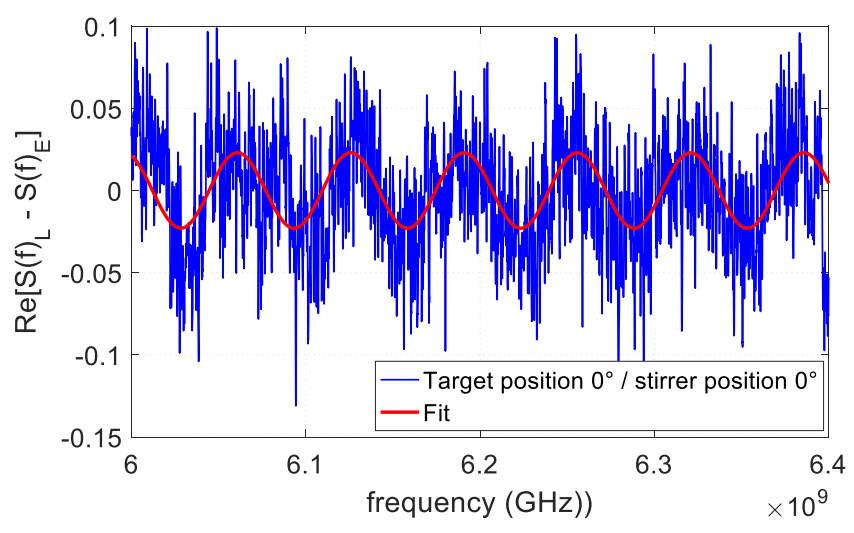

Fig. 2. Waveform versus frequency of the real part of the difference between the measured reflection coefficients of the loaded and empty cavities, at target position $0^{\circ}$ and stirrer position $0^{\circ}$.

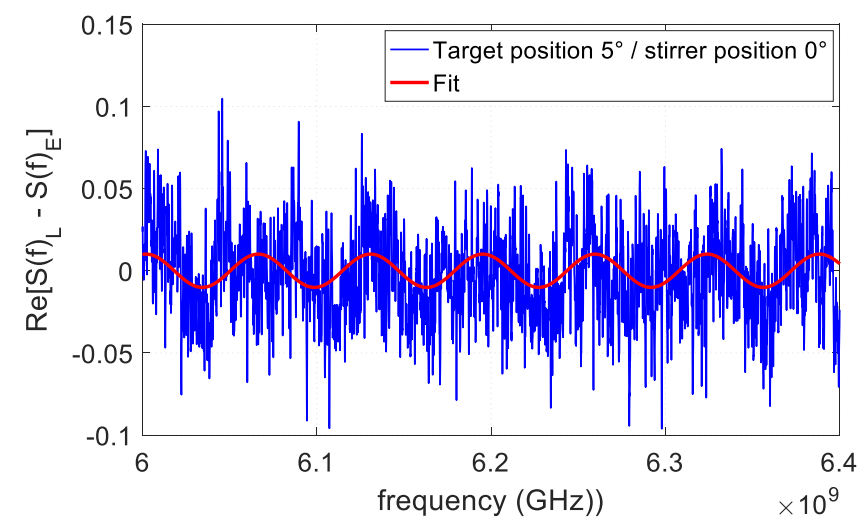

Fig. 3. Waveform versus frequency of the real part of the difference between the measured reflection coefficients for loaded and empty cavities, at target position $5^{\circ}$ and stirrer position $0^{\circ}$.

\section{RESULTS}

\section{A. Extraction of the backscattered ballistic wave according to the angle of the target}

The Fig. 2 shows the waveform when the target is orthogonal to the direct path between the antenna and the target (this target position is mentioned as the $0^{\circ}$ one), and the stirrer position is at its initial position (labelled as $0^{\circ}$ stirrer position). We notice the sine wave pattern related to the second term of (5) is partly hidden by the random signal expressed by the first term of (5). Despite this additional term, the amplitude $A$ can be extracted (red curve in Fig. 2). The amplitude of the signal related to the target backscattering appears to be constant over the considered frequency band.

The Fig. 3 corresponds to a target position of $5^{\circ}$, for which the target backscattering is of smaller amplitude than in the previous target position (Fig. 2). As a consequence, the amplitude of the signal of interest is smaller and its extraction becomes more difficult. The lack of precision in this amplitude extraction when the RCS decreases will be obvious in the following of this paper.

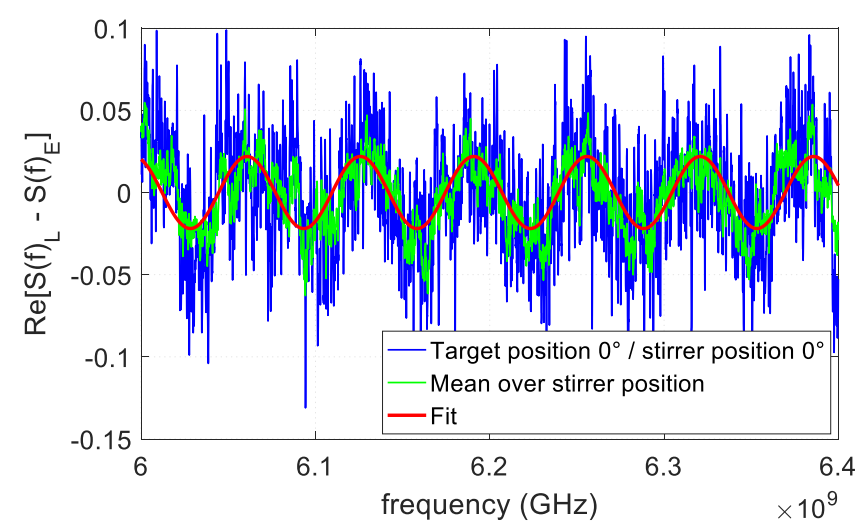

Fig. 4. Waveform versus frequency of the real part of the difference between the measured reflection coefficients of the loaded and empty cavities, at target position $0^{\circ}$. The blue curve corresponds to the stirrer position $0^{\circ}$, the green curve to the average difference over a stirrer rotation, and the red curve is the sinusoidal fitting curve.

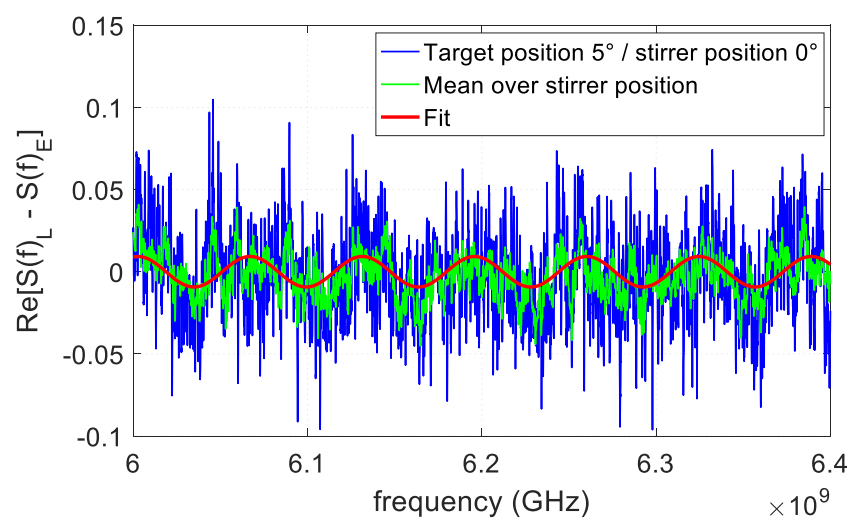

Fig. 5. Waveform versus frequency of the real part of the difference between the measured reflection coefficients for loaded and empty cavities, at target position $5^{\circ}$. The blue curve corresponds to the stirrer position $0^{\circ}$, the green curve to the average difference over a stirrer rotation, and the red curve is the sinusoidal fitting curve.

\section{B. Advantages of the mean over a stirrer rotation}

The Fig. 4 shows the average over a stirrer rotation (green curve) of the difference between both measured reflection coefficients, with and without the target. We can see that it permits to reduce the noise associated to the first term of (5) and makes easier the extraction of the sine-wave signal amplitude.

This advantage of the mode-stirring process increases for target positions related to low RCS as the low amplitude of the backscattered field makes it difficult to extract from noise. As an example for the target position of $5^{\circ}$ (Fig. 5), the sinusoidal shape of the signal of interest appears after averaging over a stirrer rotation, easing the extraction of its amplitude.

\section{Extracted Radar Cross Section pattern}

The RCS of the rectangular metallic plate has been firstly extracted for fixed stirrer positions and compared to the one obtained through simulations using ANSYS HFSS software. As no calibration measurement has been performed (using a standard target), the obtained results have been normalized so that the maximal RCS value (corresponding to the target 


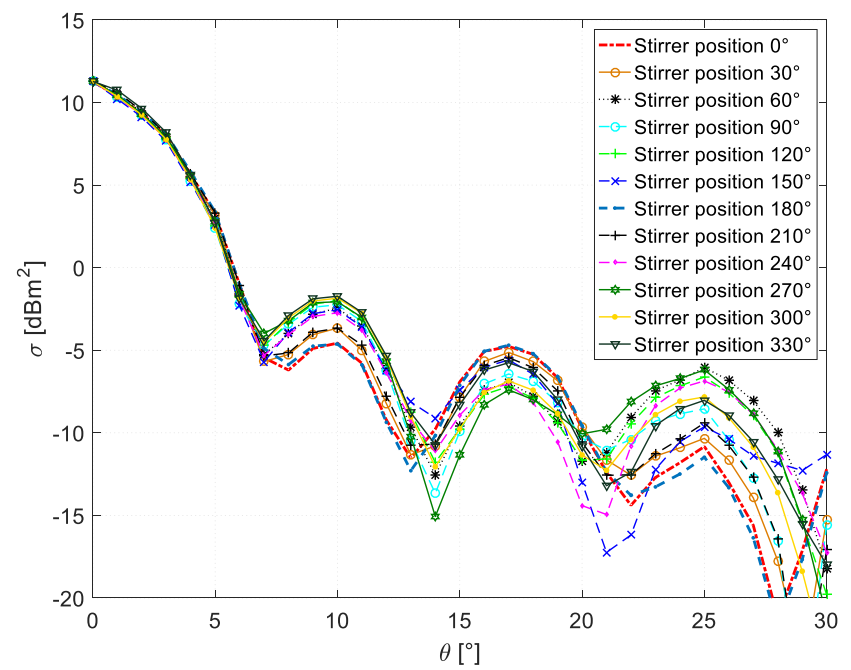

Fig. 6. RCS patterns for all stirrer positions, at a frequency of $6.2 \mathrm{GHz}$.

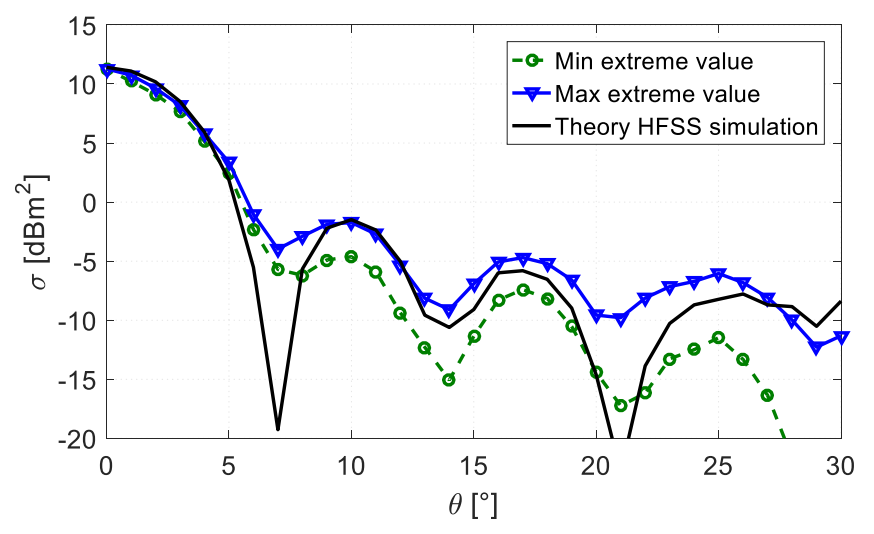

Fig. 7. Comparison between extreme RCS values among all stirrer positions and simulated RCS, at a frequency of $6.2 \mathrm{GHz}$.

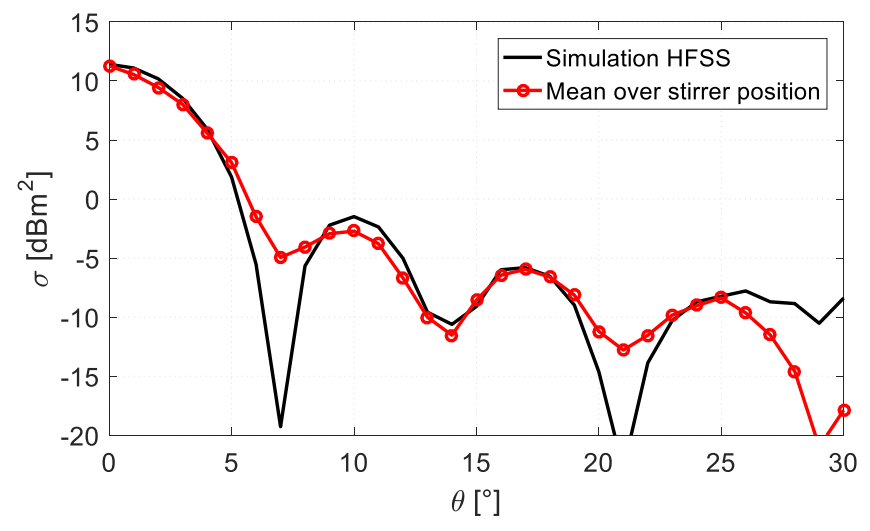

Fig. 8. Comparison between RCS pattern extracted after averaging the scattering difference over a stirrer position and simulated RCS, at a frequency of $6.2 \mathrm{GHz}$.

position $0^{\circ}$ ) is equal to the theoretical one $\sigma=\frac{4 \pi S^{2} f^{2}}{c^{2}}$ (with $S$ the target section and $c$ the light velocity). As shown in the Fig. 6 , the RCS patterns vary according to the stirrer rotation. The maximal RCS values are obtained when the target is orthogonal to the direct path between the antenna and the target (position $\theta=0^{\circ}$ ) and close to this position. In this angular range, the amplitude of the backscattering term is sufficient to permit its extraction with a good accuracy; this explains why the curves related to the different cavity configurations are superimposed for $\theta \leq 7^{\circ}$. At larger angles, the RCS decreases and becomes more difficult to extract; as a consequence, a dispersion of the results obtained with different stirrer positions is observed. The Fig. 7 presents the extreme RCS values obtained for each target position when considering all stirrer positions, and compares them to the simulation results. The theoretical RCS globally stays between these extreme values, except for very low RCS that are hard to evaluate. As shown in the previous section, averaging the difference between the measured reflection coefficients is of help to extract the RCS, in particular when the amplitude of the backscattered signal is low. This averaging technique has thus been used at each target position and results are presented in Fig. 8. It shows a good agreement between measurement and simulation except for very low RCS. Thus, this averaging approach improves the RCS estimation accuracy.

\section{CONCLUSION}

The RCS of a $20 \times 25 \mathrm{~cm}^{2}$ metallic plate is estimated at $6.2 \mathrm{GHz}$ thanks to measurement performed inside an RC. This RCS can be evaluated by retrieving the ballistic wave between the reference antenna and the target by comparing Sparameters with and without the target. This method is based on a regression process and thus is sensitive to the measurement noise. The general pattern of the RCS is well retrieved, except for target positions related to low RCS values, while compared to simulated data. However, we show that this RCS pattern strongly depends on the stirrer position during the measurement. Thus, we suggest to take benefit from the mode stirrer to average the measured S-parameters over a stirrer rotation in order to increase the overall SNR. Such process allows obtaining a much better agreement to the simulated RCS, especially for target positions related to low RCS values.

\section{ACKNOWLEDGMENT}

This work was financially supported by the French Ministry of Defence (DGA/MRIS).

\section{REFERENCES}

[1] P. V. Nikitin and K. V. S. Rao, "Theory and measurement of backscattering from RFID tags," IEEE Antennas Propag. Mag., vol. 48, no. 6, pp. 212-218, Dec. 2006.

[2] F. Sarrazin, P. Pouliguen, A. Sharaiha, J. Chauveau and P. Potier, "Antenna Physical Poles Extracted From Measured Backscattered Fields," IEEE Trans. Ant. Propag., vol. 63, no. 9, pp. 3963-3972, Sept. 2015.

[3] G. Lerosey, J. de Rosny, Scattering cross section measurement in reverberation chamber, IEEE Trans. Electromagn. Compat. vol. 49, no. 2, pp. 280-284, May 2007.

[4] C. Lemoine, E. Amador, P. Besnier, J.M. Floc'h, "Antenna directivity measurement in reverberation chamber from Rician K-factor estimation," IEEE Trans. Antennas Propagat. vol. 61, no.10, pp 53075310, Oct. 2013.

[5] P. Besnier, J. Sol and S. Meric, "Estimating Radar Cross-Section of Canonical Targets in Reverberation Chamber," EMC Europe, Angers, France, 2017. 